\title{
Bridging Game Theory and Cryptography: Recent Results and Future Directions
}

\author{
Jonathan Katz ${ }^{\star}$ \\ Department of Computer Science \\ University of Maryland \\ jkatz@cs.umd.edu
}

\begin{abstract}
Motivated by the desire to develop more realistic models of, and protocols for, interactions between mutually distrusting parties, there has recently been significant interest in combining the approaches and techniques of game theory with those of cryptographic protocol design. Broadly speaking, two directions are currently being pursued:
\end{abstract}

Applying cryptography to game theory: Certain game-theoretic equilibria are achievable if a trusted mediator is available. The question here is: to what extent can this mediator be replaced by a distributed cryptographic protocol run by the parties themselves?

Applying game-theory to cryptography: Traditional cryptographic models assume some honest parties who faithfully follow the protocol, and some arbitrarily malicious players against whom the honest players must be protected. Game-theoretic models propose instead that all players are simply self-interested (i.e., rational), and the question then is: how can we model and design meaningful protocols for such a setting?

In addition to surveying known results in each of the above areas, I suggest some new definitions along with avenues for future research.

\section{Introduction}

The fields of game theory and cryptographic protocol design are both concerned with the study of "interactions" among mutually distrusting parties. These two subjects have, historically, developed almost entirely independently within different research communities and, indeed, they tend to have a very different flavor. Recently, however, motivated by the desire to develop more realistic models of (and protocols for) such interactions, there has been significant interest in combining the techniques and approaches of both fields.

Current research at the intersection of game theory and cryptography can be classified into two broad categories: applying cryptographic protocols to gametheoretic problems, and applying game-theoretic models and definitions to the general area of cryptographic protocol design. In a bit more detail:

\footnotetext{
* Research supported in part by the U.S. Army Research Laboratory, NSF CAREER award \#0447075, and US-Israel Binational Science Foundation grant \#2004240.
} 
- Certain game-theoretic equilibria are possible if parties rely on the existence of an external trusted party called a mediator. (All the relevant definitions are given in Section 2.) This naturally motivates a cryptographen to ask: can the trusted mediator be replaced by a protocol that is run by the parties themselves? Research aimed at understanding the conditions under which the answer is positive, and developing appropriate protocols in such cases, is described in Section 3

- Traditionally, cryptographic protocols are designed under the assumption that some parties are honest and faithfully follow the protocol, while some parties are malicious and behave in an arbitrary fashion. The game-theoretic perspective, however, is that all parties are simply rational and behave in their own best interests. This viewpoint is incomparable to the cryptographic one: although no one can be trusted to follow the protocol (unless it is in their own best interests), the protocol need not prevent "irrational" behavior. The general question here is: what models and protocols are appropriate for this setting? This work is discussed in Section 4.

This paper surveys recent work in both the directions listed above, with a cryptographic audience in mind. This survey focuses more on the problems being addressed than on the solutions that have been proposed, and will thus emphasize definitions rather than concrete results. I also propose new definitional approaches to some of the problems under discussion, and have made a particular effort to highlight promising directions for future research.

Dodis and Rabin have recently written an excellent survey 16 that covers very similar ground as the present work. The present survey is perhaps a bit more technical, and somewhat more opinionated. Surveys more tangentially related to the topics considered here include those by Linial [33] and Halpern [25].

It is fascinating to observe that the recent growth of interest in blending game theory and cryptography has paralleled a surge of attention focused on game theory by computer scientists in general, most notably (for the purposes of this work) in the fields of computational complexity (see, e.g., [38, Chap. 2]), networking and distributed algorithms (see, e.g., 38, Chap. 14]), network security (see, e.g., [10] and [38, Chaps. 23, 27]), information security economics [38 Chap. 25], and more. These are all well beyond the scope of the present work.

Note: Due to space limitations, this survey has been shortened somewhat. A full version will be posted and maintained at http://eprint.iacr.org. Comments and corrections are very much appreciated.

\section{A Crash Course in Game Theory}

This section reviews some central game-theoretic concepts. I have tried to simplify things when, in my view, nothing of essence is lost (vis-a-vis the results presented here). For extensive further details, the reader is referred to [3919].

\footnotetext{
${ }^{1}$ Although, interestingly, the question was first asked in the economics community.
} 
We begin by introducing the notion of normal form games. A $n$-player game $\Gamma=\left(\left\{A_{i}\right\}_{i=1}^{n},\left\{u_{i}\right\}_{i=1}^{n}\right)$, presented in normal form, is determined by specifying, for each player $P_{i}$, a set of possible actions $A_{i}$ and a utility function $u_{i}: A_{1} \times$ $\cdots \times A_{n} \mapsto \mathbb{R}$. Letting $A \stackrel{\text { def }}{=} A_{1} \times \cdots \times A_{n}$, we refer to a tuple of actions $\boldsymbol{a}=\left(a_{1}, \ldots, a_{n}\right) \in A$ as an outcome. The utility function $u_{i}$ of party $P_{i}$ expresses this player's preferences over outcomes: $P_{i}$ prefers outcome $\boldsymbol{a}$ to outcome $\boldsymbol{a}^{\prime}$ iff $u_{i}(\boldsymbol{a})>u_{i}\left(\boldsymbol{a}^{\prime}\right)$. (We also say that $P_{i}$ weakly prefers $\boldsymbol{a}$ to $\boldsymbol{a}^{\prime}$ if $u_{i}(\boldsymbol{a}) \geq u_{i}\left(\boldsymbol{a}^{\prime}\right)$.) We assume that the $\left\{A_{i}\right\},\left\{u_{i}\right\}$ are common knowledge among the players, although the assumption of known utilities seems rather strong and it is preferable to avoid it (or assume only limited knowledge).

The game is played by having each party $P_{i}$ select an action $a_{i} \in A_{i}$, and then having all parties play their actions simultaneously. The "payoff" to $P_{i}$ is given by $u_{i}\left(a_{1}, \ldots, a_{n}\right)$ and, as noted above, $P_{i}$ is trying to maximize this value.

Two-player games (for reasonably sized $A_{1}, A_{2}$ ) can be represented conveniently in matrix form by labeling the rows (resp., columns) of the matrix with the actions in $A_{1}$ (resp., $A_{2}$ ). The entry in the cell at row $a_{1} \in A_{1}$ and column $a_{2} \in A_{2}$ contains a tuple $\left(u_{1}, u_{2}\right)$ indicating the payoffs to $P_{1}$ and $P_{2}$, respectively, given the outcome $\boldsymbol{a}=\left(a_{1}, a_{2}\right)$. For example, the following represents a game where $A_{1}=\{C, D\}, A_{2}=\left\{C^{\prime}, D^{\prime}\right\}$, and, e.g., $u_{1}\left(C, D^{\prime}\right)=1$ and $u_{2}\left(C, D^{\prime}\right)=3$ :

Table 1. A two-player game

\begin{tabular}{c|cc} 
& $C^{\prime}$ & $D^{\prime}$ \\
\hline$C$ & $(2,2)$ & $(1,3)$ \\
$D$ & $(3,1)$ & $(0,0)$
\end{tabular}

Types, and games of incomplete information. The above definition corresponds to so-called games of perfect (or complete) information. One can also consider extensions that model different features of "real-world" interactions, such as inputs provided to the parties at the beginning of the game whose values affect players' utilities. (In the game theory literature these inputs are said to determine the type of each party.) We now provide a simplified definition incorporating this situation; see 3919] for the general case.

Let $\Gamma=\left(\left\{A_{i}\right\},\left\{u_{i}\right\}\right)$ be as above, where the $\left\{u_{i}\right\}$ are now functions from $\left(\{0,1\}^{*}\right)^{n} \times A$ to the reals. Let $\mathcal{D}$ be a distribution over vectors $\left(t_{1}, \ldots, t_{n}\right)$, where each $t_{i}$ is a binary string. A game is now played as follows: first, $\left(t_{1}, \ldots, t_{n}\right)$ is sampled according to $\mathcal{D}$, and $P_{i}$ is given $t_{i}$. Next, each player $P_{i}$ plays an action $a_{i} \in A_{i}$ as before; once again, these are all assumed to played simultaneously. Then, each player $P_{i}$ receives payoff $u_{i}\left(t_{1}, \ldots, t_{n}, a_{1}, \ldots, a_{n}\right)$.

\subsection{Nash Equilibria}

If parties play a game (of perfect information), what can we expect to happen? Say $P_{1}$ knows the actions $a_{2}, \ldots, a_{n}$ that the other parties are going to 
take. Then it will choose the action $a_{1} \in A_{1}$ that maximizes $u_{1}\left(a_{1}, \ldots, a_{n}\right)$; we call this $a_{1}$ a best response of $P_{1}$ to the actions of the other players. (A best response need not be unique.) Given this action chosen by the first player, $P_{2}$ will then choose a best response $a_{2}^{\prime} \in A_{2}$, and so on. We see that a tuple $\boldsymbol{a}=\left(a_{1}, \ldots, a_{n}\right)$ is "self-enforcing" only if each $a_{i}$ represents $P_{i}$ 's best response to $\boldsymbol{a}_{-i} \stackrel{\text { def }}{=}\left(a_{1}, \ldots, a_{i-1}, a_{i+1}, \ldots, a_{n}\right)$. A tuple with this property is called a Nash equilibrium, and this serves as the starting point for all further analysis of the game. Formally, if we let $\left(a_{i}^{\prime}, \boldsymbol{a}_{i}\right)$ denote $\left(a_{1}, \ldots, a_{i-1}, a_{i}^{\prime}, a_{i+1}, \ldots, a_{n}\right)$, we have:

Definition 1. Let $\Gamma=\left(\left\{A_{i}\right\}_{i=1}^{n},\left\{u_{i}\right\}_{i=1}^{n}\right)$ be a game presented in normal form, and let $A=A_{1} \times \cdots \times A_{n}$. A tuple $\boldsymbol{a}=\left(a_{1}, \ldots, a_{n}\right) \in A$ is a (pure-strategy) Nash equilibrium if for all $i$ and any $a_{i}^{\prime} \in A_{i}$ it holds that $u_{i}\left(a_{i}^{\prime}, \boldsymbol{a}_{-i}\right) \leq u_{i}(\boldsymbol{a})$.

Another way of expressing this is to say that $a_{i} \in A_{i}$ weakly dominates $a_{i}^{\prime} \in A_{i}$ relative to $\boldsymbol{a}_{-i}$ if $u_{i}\left(a_{i}, \boldsymbol{a}_{-i}\right) \geq u_{i}\left(a_{i}^{\prime}, \boldsymbol{a}_{-i}\right)$. Then $\boldsymbol{a}$ is a Nash equilibrium if, for all $i$, the action $a_{i}$ weakly dominates all actions in $A_{i}$ relative to $\boldsymbol{a}_{-i}$.

In the example of Table $1,\left(C, D^{\prime}\right)$ is a pure-strategy Nash equilibrium: given that $P_{1}$ plays $C$, the second player prefers to play $D^{\prime}$; given that $P_{2}$ plays $D^{\prime}$, the first player prefers to play $C$. A second Nash equilibrium is given by $\left(D, C^{\prime}\right)$.

In the above definition of a pure-strategy Nash equilibrium, the "strategy" of $P_{i}$ was to deterministically play $a_{i}$ (hence the name pure strategy). If we limit players to such strategies, a Nash equilibrium may not exist in a given game. To remedy this, we allow players to follow randomized strategies as well. Specifically, if $\sigma_{i}$ is a probability distribution over $A_{i}$ then we also let $\sigma_{i}$ represent the strategy in which $P_{i}$ samples $a_{i} \in A_{i}$ according to $\sigma_{i}$ and then plays this action. (We recover deterministic strategies by letting $\sigma_{i}$ assign probability 1 to some action.) Given a strategy vector $\boldsymbol{\sigma}=\left(\sigma_{1}, \ldots, \sigma_{n}\right)$, we overload notation and let $u_{i}(\boldsymbol{\sigma})$ denote the expected utility of $P_{i}$ given that all parties play according to $\boldsymbol{\sigma}$. (We remark that although this is the standard way to assign utilities to distributions over outcomes, doing so makes the generally unrealistic assumption that players are risk neutral in that they care only about their expected utility.) The strategy $\sigma_{i}$ is a best response to $\boldsymbol{\sigma}_{-i}$ if it maximizes $u_{i}\left(\sigma_{i}, \boldsymbol{\sigma}_{-i}\right)$. Then:

Definition 2. Let $\Gamma=\left(\left\{A_{i}\right\}_{i=1}^{n},\left\{u_{i}\right\}_{i=1}^{n}\right)$ be as above, and let $\sigma_{i}$ be a distribution over $A_{i}$. Then $\boldsymbol{\sigma}=\left(\sigma_{1}, \ldots, \sigma_{n}\right)$ is a (mixed-strategy) Nash equilibrium if for all $i$ and any distribution $\sigma_{i}^{\prime}$ over $A_{i}$ it holds that $u_{i}\left(\sigma_{i}^{\prime}, \boldsymbol{\sigma}_{-i}\right) \leq u_{i}(\boldsymbol{\sigma})$.

One can verify that in the two-party game of Table 1 , the strategy vector in which $P_{1}$ plays $C$ with probability $1 / 2$, and in which $P_{2}$ plays $C^{\prime}$ with probability $1 / 2$ is a (mixed-strategy) Nash equilibrium.

The celebrated theorem of Nash [37] is that any game of perfect information where the $\left\{A_{i}\right\}$ are finite has a (mixed-strategy) Nash equilibrium. The finiteness assumption is necessary, as there are examples of two-player games with countably-infinite action sets where no mixed-strategy Nash equilibrium exists.

Nash equilibria for games of incomplete information can be defined in the natural way based on the above. Here the strategy of player $P_{i}$ corresponds to a function mapping its received input $t_{i}$ to an action $a_{i} \in A_{i}$; pure strategies 
correspond to deterministic functions. Note that here we must take into account parties' expected utilities even when considering pure-strategy Nash equilibria, since the utility of $P_{i}$ may depend on the types of the other players, and these are unknown at the time $P_{i}$ chooses its action.

\subsection{Other Equilibrium Concepts}

Nash equilibria are considered by many to be the fundamental equilibrium notion for games. Nevertheless, it is of interest to explore various refinements and strengthenings of this concept.

Dominated strategies and iterated deletion. Given game $\Gamma=\left(\left\{A_{i}\right\},\left\{u_{i}\right\}\right)$, we say that action $a_{i} \in A_{i}$ is strictly dominated with respect to $A_{-i}$ if there exists a randomized strategy $\sigma_{i} \in \Delta\left(A_{i}\right)$ such that $u_{i}\left(\sigma_{i}, \boldsymbol{a}_{-i}\right)>u_{i}\left(a_{i}, \boldsymbol{a}_{-i}\right)$ for all $\boldsymbol{a}_{-i} \in$ $A_{-i}$ (where $A_{-i} \stackrel{\text { def }}{=} \times_{j \neq i} A_{j}$ ). I.e., $a_{i}$ is strictly dominated if $P_{i}$ can always improve its situation by not playing $a_{i}$. An action $a_{i} \in A_{i}$ is weakly dominated with respect to $A_{-i}$ if there exists a randomized strategy $\sigma_{i} \in \Delta\left(A_{i}\right)$ such that $(1) u_{i}\left(\sigma_{i}, \boldsymbol{a}_{-i}\right) \geq$ $u_{i}\left(a_{i}, \boldsymbol{a}_{-i}\right)$ for all $\boldsymbol{a}_{-i} \in A_{-i}$, and (2) there exists $\boldsymbol{a}_{-i} \in A_{-i}$ such that $u_{i}\left(\sigma_{i}, \boldsymbol{a}_{-i}\right)>$ $u_{i}\left(a_{i}, \boldsymbol{a}_{-i}\right)$. I.e., $P_{i}$ can never improve its situation by playing $a_{i}$, and can sometimes improve its situation by not playing $a_{i}$.

It seems that a rational player will never choose a strictly dominated action. In fact, it is not hard to show that in any Nash equilibrium, no player assigns positive probability to any strictly dominated action. Arguably, a rational player should also never choose a weakly dominated action (although the argument in this case is less clear). If we accept this assumption, then a Nash equilibrium in which some party plays a weakly dominated action with positive probability is not expected to occur in practice. For example, consider the following game:

\begin{tabular}{c|cc} 
& $C^{\prime}$ & $D^{\prime}$ \\
\hline$C$ & $(10,10)$ & $(1,1)$ \\
$D$ & $(10,0)$ & $(2,2)$
\end{tabular}

$\left(C, C^{\prime}\right)$ is a Nash equilibrium. However, action $C$ of player $P_{1}$ is weakly dominated by action $D$. Thus, we may expect that $P_{1}$ plays $D$ - but this forces us to the Nash equilibrium $\left(D, D^{\prime}\right)$. Note that both players now end up doing worse! Intuitively, both players prefer the Nash equilibrium $\left(C, C^{\prime}\right)$, but this is not "stable" in a sense we will define below.

Say we are given a game $\Gamma^{0}$, and we have eliminated the weakly dominated actions of each player from consideration. This leaves us with "effective" action sets $\left\{A_{i}^{1}\right\}$ for each player. We may now iterate the process, and remove any actions that are weakly dominated in the "reduced game" $\Gamma^{1}=\left(\left\{A_{i}^{1}\right\},\left\{u_{i}\right\}\right)$, etc. This leads to the following definition.

Definition 3. Given $\Gamma=\left(\left\{A_{i}\right\},\left\{u_{i}\right\}\right)$ and $\hat{A} \subseteq A$, let $\mathrm{DOM}_{i}(\hat{A})$ denote the set of strategies in $\hat{A}_{i}$ that are weakly dominated with respect to $\hat{A}_{-i}$. For $k \geq 1$, set $A_{i}^{k} \stackrel{\text { def }}{=} A_{i}^{k-1} \backslash \operatorname{DOM}_{i}\left(A^{k-1}\right)$. Set $A_{i}^{\infty} \stackrel{\text { def }}{=} \cap_{k} A_{i}^{k}$. A Nash equilibrium $\boldsymbol{\sigma}$ of $\Gamma$ survives iterated deletion of weakly dominated strategies if $\sigma_{i} \in \Delta\left(A_{i}^{\infty}\right)$ for all $i$. 
Stability with respect to trembles. Another means to distinguish among a set of Nash equilibria is to ask how stable each such equilibrium is to "mistakes" (or trembles) of the other players. Such mistakes might correspond to a real mistake on the part of some player (e.g., a player chooses an irrational strategy by accident), some "out-of-band" event (e..g, a network failure), or the fact that a player's utility is slightly different than originally thought.

To define stability with respect to trembles, we must first define a metric $d$ on the strategy space $\Delta(A)$ of the players. Assuming $A$ is finite, a natural candidate is statistical difference and we assume this in the definition that follows. Various notions of stability with respect to trembles have been considered in the game theory literature, although some of them seem problematic in a cryptographic setting. The following seems best for our context:

Definition 4. Let $\Gamma=\left(\left\{A_{i}\right\},\left\{u_{i}\right\}\right)$, and let $\boldsymbol{\sigma}$ be a Nash equilibrium in $\Gamma$. Then $\sigma$ is stable with respect to trembles if there exists an $\epsilon>0$ such that for all $i$ and every $\boldsymbol{\sigma}_{-i}^{\prime} \in \Delta\left(A_{-i}\right)$ with $d\left(\boldsymbol{\sigma}_{-i}, \boldsymbol{\sigma}_{-i}^{\prime}\right)<\epsilon$, the strategy $\sigma_{i}$ is a best response to $\boldsymbol{\sigma}_{-i}^{\prime}$. I.e., for every $\sigma_{i}^{\prime} \in \Delta\left(A_{i}\right)$ it holds that $u_{i}\left(\sigma_{i}^{\prime}, \boldsymbol{\sigma}_{-i}^{\prime}\right) \leq u_{i}\left(\sigma_{i}, \boldsymbol{\sigma}_{-i}^{\prime}\right)$.

That is, even if $P_{i}$ believes there is some small probability that the other players will make a mistake (and not play according to $\boldsymbol{\sigma}_{-i}$ ), it is still in $P_{i}$ 's best interests to play according to $\sigma_{i}$.

As an example, consider the following two-player game:

\begin{tabular}{c|ccc} 
& $A^{\prime}$ & $B^{\prime}$ & $C^{\prime}$ \\
\hline$A$ & $(10,2)$ & $(1,0)$ & $(0,1)$ \\
$B$ & $(10,0)$ & $(0,0)$ & $(100,100)$
\end{tabular}

$\left(A, A^{\prime}\right)$ is a Nash equilibrium, but it is not stable with respect to trembles: if $P_{1}$ believes that $P_{2}$ might play $C^{\prime}$ with any positive probability $\epsilon$ (but still plays $B^{\prime}$ with probability 0 ), then $P_{1}$ will prefer to play $B$ rather than $A$. On the other hand, $\left(C, C^{\prime}\right)$ is a Nash equilibrium that is stable with respect to trembles: for small enough $\epsilon>0$, even if $P_{1}$ believes that $P_{2}$ might play something other than $C^{\prime}$ with probability $\epsilon$, it is still in $P_{1}$ 's best interest to play $C$.

I am not aware of any results stating conditions under which stable equilibria are guaranteed to exist.

Coalitions. Thus far, we have only been considering single-player deviations, i.e., whether it is in any single player's best interests to deviate from some prescribed strategy. Cryptographers generally prefer to think in terms of coalitions of players acting together. In general, a Nash equilibrium provides no "protection" against such coalitions.

What does it mean for a coalition $\mathcal{C}$ to prefer one outcome to another? There are at least four natural possibilities:

- $\mathcal{C}$ prefers $\boldsymbol{\sigma}$ to $\boldsymbol{\sigma}^{\prime}$ only if every player in $\mathcal{C}$ weakly prefers $\boldsymbol{\sigma}$ to $\boldsymbol{\sigma}^{\prime}$, and some player in $\mathcal{C}$ strictly prefers $\boldsymbol{\sigma}$ to $\boldsymbol{\sigma}^{\prime}$.

$-\mathcal{C}$ prefers $\sigma$ to $\sigma^{\prime}$ only if the sum of the utilities of the parties in $\mathcal{C}$ improves; i.e., if $\sum_{i \in \mathcal{C}} u_{i}(\boldsymbol{\sigma})>\sum_{i \in \mathcal{C}} u_{i}\left(\boldsymbol{\sigma}^{\prime}\right)$. (Note that for this to make sense, we must 
assume that the utility functions of all players in $\mathcal{C}$ are measured in the same units.) This definition can be viewed as capturing the ability of players in $\mathcal{C}$ to make "side payments" to each other before or after the game.

- $\mathcal{C}$ prefers $\boldsymbol{\sigma}$ to $\boldsymbol{\sigma}^{\prime}$ if any player in $\mathcal{C}$ prefers $\boldsymbol{\sigma}$ to $\boldsymbol{\sigma}^{\prime}$, i.e., if $u_{i}(\boldsymbol{\sigma})>u_{i}\left(\boldsymbol{\sigma}^{\prime}\right)$ for some $i \in \mathcal{C}$. The definition makes sense if we think of one adversarial party corrupting other parties and taking complete control over their actions. Note also that preference of $\boldsymbol{\sigma}$ to $\boldsymbol{\sigma}^{\prime}$ with respect to this definition implies preference with respect to the previous two definitions.

- Another possibility is to simply assume utility functions $u_{\mathcal{C}}$ for each possible coalition $\mathcal{C}$, and then define preference in the obvious way. This is the most general approach (it subsumes the previous three), but requires additional assumptions about players' utilities.

We adopt the third definition here.

Given a set $\mathcal{C}=\left\{i_{1}, \ldots, i_{t}\right\} \subset[n]$ and a vector $\boldsymbol{\sigma}=\left(\sigma_{1}, \ldots, \sigma_{n}\right)$, we let $A_{\mathcal{C}} \stackrel{\text { def }}{=} \times_{i \in \mathcal{C}} A_{i}, \sigma_{\mathcal{C}} \stackrel{\text { def }}{=}\left(\sigma_{i_{1}}, \ldots, \sigma_{i_{t}}\right)$, and $\sigma_{-\mathcal{C}} \stackrel{\text { def }}{=} \sigma_{[n] \backslash \mathcal{C}}$. Then:

Definition 5. Let $\Gamma=\left(\left\{A_{i}\right\}_{i=1}^{n},\left\{u_{i}\right\}_{i=1}^{n}\right)$. Then for $1 \leq t<n$ the strategy vector $\boldsymbol{\sigma}=\left(\sigma_{1}, \ldots, \sigma_{n}\right)$ is a $t$-resilient equilibrium if for all $\mathcal{C} \subset[n]$ with $|\mathcal{C}| \leq t$, all $i \in \mathcal{C}$, and any $\boldsymbol{\sigma}_{\mathcal{C}}^{\prime} \in \Delta\left(A_{\mathcal{C}}\right)$, it holds that $u_{i}\left(\boldsymbol{\sigma}_{\mathcal{C}}^{\prime}, \boldsymbol{\sigma}_{-\mathcal{C}}\right) \leq u_{i}(\boldsymbol{\sigma})$.

That is, for every coalition $\mathcal{C}$ of size at most $t$, no member of the coalition improves its situation no matter how the members of $\mathcal{C}$ coordinate their actions.

Observe that a 1-resilient equilibrium is a Nash equilibrium. Extending other equilibrium concepts to the case of coalitions seems not to have been explored significantly.

Mixed models. It is standard in game theory to assume that all players are rational. Recent work [1/35/2] has explored models where most parties are rational, but some players are malicious and behave arbitrarily. Treating players as malicious can be viewed (to some extent) as treating their utilities as completely unknown. It is also possible to assume that some players honestly follow the proscribed protocol - perhaps out of altruism or laziness - rather than seeking to improve their utility (although it should be in these players' interests to run the protocol altogether). These are interesting directions that are not discussed any further in this survey.

\subsection{Correlated Equilibria}

Correlated equilibria [3] offer another solution concept with some advantages relative to Nash equilibria. In some games, there may exist a correlated equilibrium that, for every party $P_{i}$, gives a better payoff to $P_{i}$ than any Nash equilibrium (see [36] for an example). More generally, correlated equilibria have payoffs outside the convex hull of all Nash equilibria, and therefore give more options to the players. Finally, correlated equilibria of any game can be computed in polynomial time, something not believed to be the case for Nash equilibria. 
Given a game $\Gamma=\left(\left\{A_{i}\right\},\left\{u_{i}\right\}\right)$, we define a mediated version of $\Gamma$ which relies on a trusted, external party $M$ called the mediator. The game is now played in two stages: first, the mediator chooses a vector of actions $\boldsymbol{a} \in A$ according to some known distribution $\mathcal{M}$, and then hands the recommendation $a_{i}$ to player $P_{i}$. The players then play $\Gamma$ as before by choosing any action in their respective action sets. Players are "supposed" to follow the recommendation of the mediator, and a correlated equilibrium is one in which it is in each player's best interests to do so. To formally define this notion, let $u_{i}\left(a_{i}^{\prime}, \boldsymbol{a}_{-i} \mid a_{i}\right)$ denote the expected utility of $P_{i}$, given that it plays action $a_{i}^{\prime}$ after having received recommendation $a_{i}$ and all other parties play their recommended actions $\boldsymbol{a}_{-i}$. (The expectation here is over $\boldsymbol{a}$ sampled according to $\mathcal{M}$.)

Definition 6. Let $\Gamma=\left(\left\{A_{i}\right\},\left\{u_{i}\right\}\right)$. A distribution $\mathcal{M} \in \Delta(A)$ is a correlated equilibrium if for all $\boldsymbol{a}=\left(a_{1}, \ldots, a_{n}\right)$ in the support of $\mathcal{M}$, all $i$, and all $a_{i}^{\prime} \in A_{i}$, it holds that $u_{i}\left(a_{i}^{\prime}, \boldsymbol{a}_{-i} \mid a_{i}\right) \leq u_{i}\left(\boldsymbol{a} \mid a_{i}\right)$.

Any Nash equilibrium is a correlated equilibrium, but Nash equilibria correspond to the special case where $\mathcal{M}$ is a product distribution over the $A_{i}$.

Let $u_{i}(\mathcal{M})$ denote the expected utility of $P_{i}$ when all parties follow their actions as recommended by $\mathcal{M}$. A definition equivalent to the previous one, but better suited for extensions to coalitions as well as the computational setting, is:

Definition 7. Let $\Gamma=\left(\left\{A_{i}\right\},\left\{u_{i}\right\}\right)$. A distribution $\mathcal{M} \in \Delta(A)$ is a correlated equilibrium if for all $i$ and any $f_{i}: A_{i} \rightarrow A_{i}$ it holds that

$$
u_{i}\left(f_{i}\left(a_{i}\right), \boldsymbol{a}_{-i}\right) \leq u_{i}(\mathcal{M}),
$$

where $\boldsymbol{a}$ is sampled according to $\mathcal{M}$.

As an example of a game with a correlated equilibrium that is not a Nash equilibrium, consider the two-party game of Table 1 and the distribution that assigns probability $1 / 3$ to each of $\left(C, D^{\prime}\right),\left(D, C^{\prime}\right)$, and $\left(C, C^{\prime}\right)$. One can check that neither party has any incentive to deviate from their recommended action, and each player has expected utility 2 (an improvement on the mixed-strategy Nash equilibrium described in Section 2.1).

In games of incomplete information (as we have defined them in Section 2), a mediated game is played as follows: first, a vector $\left(t_{1}, \ldots, t_{n}\right)$ is sampled according to a distribution $\mathcal{D}$, and $t_{i}$ is given to $P_{i}$. Then, each party $P_{i}$ sends some $t_{i}^{\prime}$ to the mediator. Based on the vector $\boldsymbol{t}^{\prime}=\left(t_{1}^{\prime}, \ldots, t_{n}^{\prime}\right)$ received, the mediator samples a vector $\boldsymbol{a} \in A$ according to a distribution $\mathcal{M}\left(\boldsymbol{t}^{\prime}\right)$, and recommends action $a_{i}$ to player $P_{i}$. The parties then play as before, choosing whether or not to follow the mediator's recommendation. Correlated equilibria in this situation are defined as the natural extension of the above, through we stress that a player's strategy now determines both what value $t_{i}^{\prime}$ it sends to the mediator (as a function of the received input $t_{i}$ ) as well as what action it plays in the game. A correlated equilibrium is said to be truthful if it is in each party's best interest to send $t_{i}^{\prime}=t_{i}$ to the mediator. The revelation principle characterizes when truthful correlated equilibria exist. 
Correlated equilibria in the presence of coalitions. The basic approach used to handle coalitions in Definition 5 can be extended in the natural way to the case of correlated equilibria. Two variants of the definition are obtained, however, depending on the details of how the mediated game is played. If ex ante collusion is allowed, the parties in a coalition $\mathcal{C}$ may coordinate their strategies in advance, but are assumed unable to communicate after the mediator provides them with their recommended actions. If ex post collusion is allowed, the parties in $\mathcal{C}$ can communicate even after receiving their recommendations from the mediator.

Definition 8. Let $\Gamma=\left(\left\{A_{i}\right\},\left\{u_{i}\right\}\right)$ be an $n$-party game, and let $1 \leq k<n$. $A$ distribution $\mathcal{M} \in \Delta(A)$ is an ex ante $t$-resilient correlated equilibrium if for all $\mathcal{C} \subset[n]$ with $|\mathcal{C}| \leq k$, any functions $\left\{f_{i}: A_{i} \rightarrow A_{i}\right\}_{i \in \mathcal{C}}$, and all $i \in \mathcal{C}$ it holds that $u_{i}\left(\left\{f_{i}\left(a_{i}\right)\right\}_{i \in \mathcal{C}}, \boldsymbol{a}_{-\mathcal{C}}\right) \leq u_{i}(\mathcal{M})$, where $\boldsymbol{a}$ is sampled according to $\mathcal{M}$.

$\mathcal{M}$ is an ex post $t$-resilient correlated equilibrium if for all $\mathcal{C}$ as above, any function $f_{\mathcal{C}}: A_{\mathcal{C}} \rightarrow A_{\mathcal{C}}$, and any $i \in \mathcal{C}$ it holds that $u_{i}\left(f_{\mathcal{C}}\left(\boldsymbol{a}_{\mathcal{C}}\right), \boldsymbol{a}_{-\mathcal{C}}\right) \leq u_{i}(\mathcal{M})$, where $\boldsymbol{a}$ is sampled according to $\mathcal{M}$.

\subsection{Extensive Form Games}

Extensive form games remove the assumption that players act simultaneously. Such games are best described as occurring in a sequence of rounds, where in any given round the game might specify that all parties play simultaneously (as in a normal form game) or that some subset of designated parties plays. Play of the game thus defines a history of the actions taken by the players thus far, and a player $P_{i}$ 's strategy $\sigma_{i}$ now specifies, for each round in which it is $P_{i}$ 's turn to move, a (randomized) function mapping possible histories to actions. Players' utilities are now functions of terminal histories (i.e., histories that occur at the end of the game), rather than functions of the strategy vector of the players. We rely on the above intuitive description rather than present a formal definition.

We provide a simple example of an extensive form game, which also demonstrates how introducing alternation can affect the outcome of a game. Consider a seller $P_{1}$ and a buyer $P_{2}$, where $P_{1}$ can either sell high $(H)$ or low $(L)$, and $P_{2}$ can choose either to buy $(B)$ or not $(N)$. Payoffs are given by the matrix on the left, but we will assume that the seller announces its action first. This gives an extensive form game in which the buyer can follow any of four (pure) strategies; we let $X Y$ denote the strategy where $P_{2}$ chooses $X$ if the seller chooses $H$, and $P_{2}$ chooses $Y$ if the seller chooses $L$. This extensive form game is represented in normal form in the matrix on the right.

Table 2. An extensive form game presented in normal form

\begin{tabular}{c|cc} 
& $B$ & $N$ \\
\hline$H$ & $(10,1)$ & $(0,0)$ \\
$L$ & $(5,6)$ & $(0,0)$
\end{tabular}

\begin{tabular}{c|cccc} 
& $B B$ & $B N$ & $N B$ & $N N$ \\
\hline$H$ & $(10,1)$ & $(10,1)$ & $(0,0)$ & $(0,0)$ \\
$L$ & $(5,6)$ & $(0,0)$ & $(5,6)$ & $(0,0)$
\end{tabular}


Looking at the game on the right, we see that $(L, N B)$ is a Nash equilibrium. The strategy being followed by $P_{2}$ is to refuse to buy if the buyer charges the higher price, and if the seller knows that $P_{2}$ will follow this strategy then it is in the seller's best interest to charge a low price. In contrast, the game on the left (where parties move simultaneously) has the unique Nash equilibrium $(H, B)$.

Something odd about the Nash equilibrium $(L, N B)$ of the extensive form game is that $P_{2}$ is, in essence, threatening to play irrationally if $P_{1}$ plays $H$ (since, conditioned on $P_{1}$ playing $H$, the buyer is better off playing $B$ than $N$ ). Another way to say this is that $P_{2}$ plays rationally given any realizable history (where history $h$ is realizable with respect to $\sigma$ if this history occurs with positive probability when all parties play according to $\boldsymbol{\sigma}$ ), but $P_{2}$ threatens to play irrationally at some non-realizable history. In the following section, we will discuss a refinement of Nash equilibria that eliminates such "empty threat" strategies.

\subsection{Equilibrium Concepts in Extensive Form Games}

Any game in extensive form can be viewed as a normal form game by letting the set of allowable actions correspond to the players' strategies. Thus, all the equilibrium concepts we have discussed previously can be applied to extensive form games as well. However, it is often more natural to view certain games in extensive form, and thinking of games in this way motivates new equilibrium concepts. In particular, a question that arises with regard to extensive form games is whether we need to "pay attention" to players' strategies at non-realizable histories. In some cases paying attention to such strategies makes intuitive sense, while in other cases the situation is less clear.

Subgame perfect equilibria. As noticed in the previous section, certain strategy vectors may be Nash equilibria but contain "empty threats" by one or more of the players. Subgame perfect Nash equilibria eliminate this possibility. To define this concept, we introduce (informally) the notion of the reduced game $\Gamma^{h}$ of an extensive form game $\Gamma$. Basically, $\Gamma^{h}$ corresponds to $\Gamma$ where some initial history $h$ is fixed; we may view $\Gamma^{h}$ as the continuation of $\Gamma$ conditioned on the fact that history $h$ has been observed thus far. A strategy $\sigma_{i}$ in $\Gamma$ naturally induces a strategy $\sigma_{i}^{h}$ in $\Gamma^{h}$ by setting $\sigma_{i}^{h}\left(h^{\prime}\right)=\sigma_{i}\left(h \| h^{\prime}\right)$.

Definition 9. Let $\Gamma$ be an extensive form game, and let $\boldsymbol{\sigma}$ be a Nash equilibrium in $\Gamma$. Then $\boldsymbol{\sigma}$ is subgame perfect if for all possible histories $h$ of $\Gamma$, the strategy vector $\boldsymbol{\sigma}^{h}$ is a Nash equilibrium of the reduced game $\Gamma^{h}$.

Recall that a history $h$ is realizable (with respect to $\boldsymbol{\sigma}$ ) if it occurs with positive probability when all parties follow $\boldsymbol{\sigma}$. If the definition above only quantified over realizable histories, then every Nash equilibrium would satisfy the definition.

In the game of Table 2 the Nash equilibrium $(L, N B)$ is not subgame perfect because, conditioned on the (non-realizable) history in which $P_{1}$ plays $H$, player $P_{2}$ prefers to play $B$ instead of $N$. Equilibrium $(H, B B)$ is subgame perfect. 
Stability with respect to trembles. There are two possible ways to extend the definition of stability with respect to trembles to extensive form games, depending on whether or not subgame perfection is also required. The following definition does not take subgame perfection into account. Say two strategies $\sigma_{i}, \sigma_{i}^{\prime}$ of $P_{i}$ yield equivalent play with respect to $\sigma$ if for every history $h$ realizable with respect to $\boldsymbol{\sigma}$ it holds that $\sigma_{i}(h)=\sigma_{i}^{\prime}(h)$. (This just means that, assuming all other parties play $\boldsymbol{\sigma}_{-i}$, play proceeds identically whether $P_{i}$ plays $\sigma_{i}$ or $\sigma_{i}^{\prime}$.)

Definition 10. Let $\Gamma$ be an extensive form game, and let $\boldsymbol{\sigma}$ be a Nash equilibrium in $\Gamma$. Then $\sigma$ is stable with respect to trembles (for realizable histories) if there exists an $\epsilon>0$ such that for all $i$ and every $\boldsymbol{\sigma}_{-i}^{\prime}$ with $d\left(\boldsymbol{\sigma}_{-i}, \boldsymbol{\sigma}_{-i}^{\prime}\right)<\epsilon$ there exists a $\sigma_{i}^{\prime}$ that is a best response to $\sigma_{-i}^{\prime}$ and such that $\sigma_{i}$ and $\sigma_{i}^{\prime}$ yield equivalent play with respect to $\boldsymbol{\sigma}$.

\subsection{Cryptographic Considerations}

In a cryptographic setting, it is natural to modify the way games are treated and the way various equilibrium notions are defined. We give an example of how this might be done for the specific case of parties running a protocol in the standard cryptographic sense, though it can be easily extended for more general scenarios (for examples, parties running a protocol and then taking some action as in Section 3. or parties who receive some initial input as in Section 4).

As usual in the cryptographic setting, we introduce a security parameter $k$ provided to all parties at the beginning of the game. The action of a player $P_{i}$ now corresponds to running an interactive Turing machine (ITM) $M_{i}$. This ITM $M_{i}$ takes as input some current state and incoming messages from the other parties, and outputs the next message of player $P_{i}$ along with updated state. The message $m_{i}$ is then sent to all other parties (we are assuming here that communication is over a broadcast channel). We require $M_{i}$ to run in probabilistic polynomialtime, which we take to mean that the next message function is computed in time polynomial in $k$. This definition allows $M_{i}$ to run for an unbounded number of rounds and, if desired, we can additionally require that the expected number of rounds for which $M_{i}$ runs is also polynomial.

Utility functions take the security parameter $k$ as input, and are functions mapping transcripts of a protocol execution to the reals that can be computed in time polynomial in $k$. We stress that, as in extensive form games, utilities depend only on the "observable outcome" of the game play.

For the purposes of this section, we define a computational game $\Gamma$ to be one in which the actions of each player correspond to the set of probabilistic polynomial-time ITMs, and where the utilities of each player are polynomialtime computable. We remark that we no longer need to consider mixed strategies, since a mixed strategy that can be implemented in polynomial time corresponds to a pure strategy (since pure strategies correspond to randomized ITMs).

An important difference between the cryptographic setting and the setting we have considered until now is that now parties are assumed to be indifferent to negligible changes in their utilities. For example: 
Definition 11. Let $\Gamma=\left(\left\{A_{i}\right\},\left\{u_{i}\right\}\right)$ be a computational game. A strategy vector $\boldsymbol{M}=\left(M_{1}, \ldots, M_{n}\right)$ is a computational Nash equilibrium if for all $i$ and any probabilistic polynomial-time ITM $M_{i}^{\prime}$ there is a negligible function $\epsilon$ such that

$$
u_{i}\left(k, M_{i}^{\prime}, M_{-i}\right)-u_{i}(k, M) \leq \epsilon(k) .
$$

I am unaware of any result characterizing conditions under which Nash equilibria exist in computational games.

Subgame perfection and related notions. Execution of a protocol can naturally be regarded as an extensive form game. Extending equilibrium notions for extensive form games to the computational setting is, however, less obvious. For example, a first approach to extending the notion of subgame perfection to the computational setting would be to say that the strategy vector $\boldsymbol{\sigma}$ of the game $\Gamma$ is subgame perfect if for all possible histories $h$ of $\Gamma$, the strategy vector $\sigma^{h}$ is a computational Nash equilibrium of the reduced game $\Gamma^{h}$. However, this ignores the probability with which history $h$ is reached! On the other hand, it is unclear how to assign a probability to a non-realizable history. We are not aware of any definition of computational subgame perfection that deals with these issues.

A recent definition suggested by Kol and Naor [29] explicitly rejects the idea of "weighting" the utility of strategies according to the probability with which a given history is reached. Instead, informally, they require that conditioned on reaching any history that occurs with positive probability, players' strategies should remain in equilibrium. In their definition of a computational game, they allow players to use ITMs which run in time polynomial in $k+r$, where $r$ is the number of rounds that have been played thus far. (Thus, the next-message function in their case may be viewed as a function from the entire history/transcript thus far to a next message, rather than from some internal state and a set of incoming messages to a next message, as defined above.) For lack of any better name, we refer to ITMs of this sort as running in liberal polynomial time and refer to the notion of $t$-resilient* equilibria for strategy vectors that remain in equilibrium even with respect to this stronger class of machines. Finally, we let $u_{i}(\cdot \mid h)$ denote the expected utility of $P_{i}$ conditioned on history $h$. We now give the definition of Kol and Naor 2

Definition 12. Let $\Gamma$ be a computational game. A strategy vector $\boldsymbol{M}=\left(M_{1}\right.$, $\left.\ldots, M_{n}\right)$ is computationally $t$-immune 3 if for every history $h$ realizable with respect to $\boldsymbol{M}$ and every $i$, the strategy vector $\boldsymbol{M}^{h}$ is a t-resilient ${ }^{*}$ Nash equilibrium in the reduced game $\Gamma^{h}$. I.e., for every $\mathcal{C} \subset[n]$ with $|\mathcal{C}| \leq t$ and every liberal polynomial-time ITM $M_{\mathcal{C}}^{\prime}$ there is a negligible function $\epsilon$ such that

$$
u_{i}\left(k, M_{\mathcal{C}}^{\prime}, M_{-\mathcal{C}} \mid h\right)-u_{i}(k, M \mid h) \leq \epsilon(k) .
$$

\footnotetext{
${ }^{2}$ One change we introduce is to condition on observable histories rather than on players' random coins (which may be private).

3 Note that immunity refers to an entirely different concept in 1 .
} 


\subsection{Critiques of Game Theory}

Without going into much detail here, I will simply say that it is not at all clear whether game theory provides the "best" way of modeling interactions, both in general as well as specifically in a cryptographic setting. (All of the critiques I mention here are well-known, and not in any way novel.) For starters, it is unclear the extent to which the behavior of most people can be modeled as rational. (Social economists study exactly this issue.) Even if we are willing to believe that people act rationally, it is not always clear when a protocol designer can assume any knowledge of their utilities.

Irrespective of the above, many of the solution concepts are unsatisfying. The notion of a Nash equilibrium is perhaps the most intuitively appealing one, but in cases where multiple Nash equilibria exist it is unclear which one the parties will settle on or even if they can agree to settle on one at all. Other notions have been introduced in an effort to distinguish among various Nash equilibria, but it seems that for every such notion there exists a game in which applying the notion goes against one's intuition. (See, e.g., [19, pp. 462-463] for an example in the context of iterated deletion of weakly dominated strategies where it is to one party's advantage to publicly burn their money.)

\section{Implementing Mediators Using Cryptography}

As we have seen in Section 2.3, if parties are willing to assume the existence of a trusted mediator then they can potentially achieve certain equilibria that may be "preferable" to any of the available Nash equilibria. If a trusted mediator is not available, the question becomes: to what extent can the parties themselves run a protocol in place of the mediator?

This question was first explored in the economics community [14]6/18|9|42]43|4 (see 2] for a summary of these results), where researchers suggested "cheap talk" protocols by which parties could communicate amongst themselves to implement a correlated equilibrium. (As the terminology suggests, communication among the players is "cheap" in the sense that it costs nothing; it is also "worth nothing" in the sense that players are not "bound" to any statements they make; e.g., there is no legal recourse if someone lies). In the cryptography community, the question was first addressed by Dodis, Halevi, and Rabin [15].

\subsection{Defining the Problem}

Let us begin by defining the basic problem. (Other variants and extensions will be explored below.) We are given some $n$-party game $\Gamma=\left(\left\{A_{i}\right\},\left\{u_{i}\right\}\right)$ in normal form, along with a correlated equilibrium $\mathcal{M}$. We then define the extensive form game $\Gamma_{C T}$ in which all parties hold a common security parameter $k$ and first communicate in a "cheap talk" phase. The parties then play $\Gamma$, making their moves simultaneously (as always). Following the game-theoretic convention, all parties must play some action in $\Gamma$. (I.e., we do not allow player $P_{i}$ to "abort" in 
$\Gamma$ unless this is an action in $A_{i}$.) On the other hand, following the cryptographic convention we do allow players to abort (and refuse to send any more messages) during the cheap talk phase.

We make no assumptions regarding the exact communication model during the cheap talk phase. For now, however, we assume that colluding parties can communicate "out of band" throughout the entire game. (This assumption is removed in Section 3.4.) Thus, for now we focus on ex post correlated equilibria which are resilient to coalitions even when such communication is allowed.

A player's strategy in $\Gamma_{C T}$ determines both the protocol it runs in the cheap talk phase as well as the action it plays in $\Gamma$. We may now define the basic goal:

Definition 13. Let $\Gamma$ be a game, and let $\mathcal{M}$ be an ex post t-resilient correlated equilibrium in $\Gamma$. Let $\Gamma_{C T}$ be the cheap talk extension of $\Gamma$, and let $\boldsymbol{\sigma}$ be an efficient strategy vector in $\Gamma_{C T}$. Then $\boldsymbol{\sigma}$ is a t-resilient implementation of $\mathcal{M}$ if (1) $\boldsymbol{\sigma}$ is a t-resilient computational equilibrium in $\Gamma_{C T}$, and (2) for all $i$, it holds that $u_{i}(k, \boldsymbol{\sigma})=u_{i}(k, \mathcal{M})$.

One might strengthen the definition to require that the distribution of payoffs in $\Gamma_{C T}$ (both for each party as well as when considering joint distributions among multiple parties) is close to the distribution of payoffs in the original mediated game. A stronger requirement of a different flavor is given by Lepinski et al. [30], who require (informally) that any vector of expected payoffs achievable by $\mathcal{C}$ in $\Gamma_{C T}$ (i.e., even ones that are sub-optimal for $\mathcal{C}$ ) can also be achieved by $\mathcal{C}$ in the original mediated game. We do not impose such requirements here.

\subsection{A Simple Observation}

It is instructive to begin with a relatively simple observation: if $t, n$, and the communication model are such that completely fair secure multi-party computation [20, Def. 7.5.4] is possible, then any correlated equilibrium $\mathcal{M}$ of any game $\Gamma$ has a $t$-resilient implementation: During the cheap talk phase the parties run a completely fair protocol $\Pi$ computing $\boldsymbol{a} \leftarrow \mathcal{M}$, where $P_{i}$ receives $a_{i}$ as output. Following the cheap talk phase, each party plays the action it received as output in $\Pi$. It is not hard to see that the strategy vector thus specified (i.e., "run $\Pi$ and then play the result") is a $t$-resilient (computational) equilibrium with expected payoffs identical to those in the original mediated game.

Applying the above observation to the standard communication model, we see that if parties are connected by pairwise point-to-point channels then a $t$-resilient implementation of any correlated equilibrium exists when $t<n / 3$. If a broadcast channel or a PKI is additionally assumed, then $t$-resilient implementations exist whenever $t<n / 2$. The above all follow from standard results in secure multi-party computation [118,407]. Lepinski et al. 30. show how to achieve completely fair secure computation for any $t<n$ - and hence show $t$-resilient implementations of any correlated equilibrium for $t<n-$ in a non-standard communication model where "secure envelopes" are assumed. (Completely fair secure multi-party computation using point-to-point channels and broadcast is, 
in general, impossible for $t \geq n / 2$ [13].) Assuming secure envelopes may be reasonable in some settings, but such envelopes seem impossible to realize (without assuming trusted parties) in a distributed setting such as the Internet.

\subsection{Implementing Mediators without Completely Fair MPC}

The natural next question is: when can a correlated equilibrium be implemented even though completely fair secure computation is ruled out? The initial result in this direction is due to Dodis, Halevi, and Rabin 15, who examine the case $t=1, n=2$. Before explaining their solution, we first introduce some terminology. Let $\Gamma$ be a game in normal form. Then the minimax profile against player $P_{i}$ is an action $\boldsymbol{a}_{-i} \in A_{-i}$ (or, more generally, in the product distribution $\left.\times_{j \neq i} \Delta\left(A_{i}\right)\right)$ minimizing $\max _{a_{i} \in A_{i}}\left\{u_{i}\left(a_{i}, \boldsymbol{a}_{-i}\right)\right\}$. In other words, a minimax profile $\boldsymbol{a}_{-i}$ "punishes" $P_{i}$ by giving $P_{i}$ its lowest possible utility, assuming $P_{i}$ plays a best response to the strategy of the other parties.

The basic idea of Dodis, Halevi, and Rabin is as follows: Let $\mathcal{M}$ be a correlated equilibrium in some two-party game $\Gamma$. In $\Gamma_{C T}$, the two parties run a protocol $\Pi$ for computing $\left(a_{1}, a_{2}\right) \leftarrow \mathcal{M}$, where party $P_{i}$ receives $a_{i}$ as output. This protocol $\Pi$ is "secure-with-abort" (cf. [20, Def. 7.2.6]), which informally means that privacy and correctness hold but fairness does not; in particular, we assume it is possible for $P_{1}$ to receive its output even though $P_{2}$ does not. After running $\Pi$, each party plays the action it received as output in $\Pi$; if $P_{2}$ does not receive output from $\Pi$ then it plays the minimax profile against $P_{1}$.

It is not hard to see that this is a 1-resilient implementation of $\mathcal{M}$. First, it is immediate that if both parties play the indicated strategy, then the payoffs of both parties in $\Gamma_{C T}$ are exactly the payoffs they would receive by playing $\mathcal{M}$ in $\Gamma$. Let us now argue that this is a computational Nash equilibrium in $\Gamma_{C T}$. We first observe that $P_{2}$ has no incentive to deviate: no matter how $P_{2}$ plays when running $\Pi$, party $P_{1}$ receives correctly-distributed output and plays according to the correlated equilibrium. Given this, $P_{2}$ 's best action to play in $\Gamma$ is given by its own output from $\Pi$. We remark that here we are relying on the assumption that $P_{2}$ can only run polynomial-time strategies, and that $P_{2}$ is indifferent to negligible differences in its expected utility, exactly as we have defined things in Definition 11 .

As for $P_{1}$, the only way it can (effectively) deviate during the cheap talk phase is by running $\Pi$ until it receives its own output $a_{1}$ and then possibly aborting the protocol so that $P_{2}$ does not receive any output. We claim that it is never to $P_{1}$ 's advantage to abort. (Note that the analysis in [15] seems to assume that $P_{1}$ either never aborts or always aborts, but of course $P_{1}$ can determine whether to abort based on its output.) If $P_{1}$ allows $P_{2}$ to receive its output, this induces some mixed strategy $\sigma_{2}$ that will be played by $P_{2}$. (I.e., $\sigma_{2}$ represents the marginal distribution on $P_{2}$ 's recommended action according to $\mathcal{M}$, conditioned on the fact that $P_{1}$ 's recommended action is $a_{1}$.) Since $\mathcal{M}$ is a correlated equilibrium, $a_{1}$ is a best response to $\sigma_{2}$. If $P_{1}$ aborts, then $P_{2}$ will play a minimax profile $\sigma_{2}^{\prime}$ against $P_{1}$. By definition of a minimax profile, $P_{1}$ 's best response to $\sigma_{2}^{\prime}$ cannot give $P_{1}$ better utility than its best response to $\sigma_{2}$. 
We conclude that $P_{1}$ always does worse by aborting the execution of $\Pi$. Given that both parties receive output in $\Pi$, it is obviously to $P_{1}$ 's advantage to play its recommended action. This completes the proof.

Extensions. The above ideas do not extend easily to a more general setting. For example, consider the case $t=1, n=3$ (with point-to-point communication). If these parties run a protocol $\Pi$ in which a single deviating party can abort the computation without being identified, then the remaining parties do not know which player to "punish". In fact, essentially this situation is inherent in general [2, Theorem 4]. On the other hand, specific correlated equilibria may be implementable using the general approach discussed below.

Next look at the case $t=2, n<5$. Observe that even if one party, say $P_{1}$, is identified as cheating, the naive approach of having the remaining parties play a minimax profile against $P_{1}$ may not work. For one thing, although such a profile might result in a worse payoff for $P_{1}$, it may actually lead to a better payoff for a second player, say $P_{2}$, colluding with $P_{1}$. (And recall that 2 -resilience only holds if deviations help no one in the coalition.) Moreover, if players play a minimax profile against $P_{1}$, it may be possible for $P_{2}$ (who, recall, is colluding with $P_{1}$ ) to deviate from the minimax profile and thus benefit $P_{1}$.

We are thus motivated to define a stronger notion of "punishment". Following [1, Def. 5], though differing in some respects, we define:

Definition 14. Let $\Gamma$ be an n-party game with correlated equilibrium $\mathcal{M}$. $A$ strategy vector $\boldsymbol{\sigma}$ is a $t$-punishment strategy with respect to $\mathcal{M}$ if for all $\mathcal{C} \subset[n]$ with $|\mathcal{C}| \leq t$, all $\boldsymbol{\sigma}_{\mathcal{C}}^{\prime}$, and all $i \in \mathcal{C}$ it holds that $u_{i}\left(\boldsymbol{\sigma}_{\mathcal{C}}^{\prime}, \boldsymbol{\sigma}_{-\mathcal{C}}\right) \leq u_{i}(\mathcal{M})$.

That is, any coalition would be better off following the recommendations of $\mathcal{M}$ rather than playing against $\boldsymbol{\sigma}_{-\mathcal{C}}$.

If a $t$-punishment strategy is available for a given correlated equilibrium $\mathcal{M}$, then this gives hope that a variant of the Dodis-Halevi-Rabin approach can be used to give a $t$-resilient implementation of $\mathcal{M}$. See 12 for work along these lines. Also relevant is the work of $3127 / 28$, discussed in more detail in the following section. As we have mentioned earlier, a partial converse [2] of the positive result just mentioned shows that, in general, if a $t$-punishment strategy is not available for a given correlated equilibrium $\mathcal{M}$, then this equilibrium cannot be implemented. Further work is need to better characterize the exact conditions under which a given correlated equilibrium can or cannot be implemented.

\subsection{Implementing ex ante Equilibria (and More)}

This section provides a brief discussion of work aimed at a slightly different aspect of the problem. Assume now that colluding parties cannot communicate "out of band" once $\Gamma_{C T}$ begins; i.e., during the cheap talk phase of $\Gamma_{C T}$ all communication is done over a public channel, and after the cheap talk phase when it is time for the parties to play $\Gamma$ - there is no inter-party communication at all. (Colluding parties can try to communicate over the broadcast channel, 
but if they are obvious about it then this will be detected by the other parties and punished.) It is then meaningful to ask whether it is possible to implement an ex ante correlated equilibrium of $\Gamma$ in the cheap talk extension $\Gamma_{C T}$.

This problem is not immediately solved even if completely fair secure computation is possible. The problem is that covert channels may exist in the protocol itself. If such covert communication is possible, an ex ante correlated equilibrium may no longer remain an equilibrium. Informally, say a protocol is collusion free if covert communication is impossible. (We remark, however, that it seems sufficient here to prevent covert communication only after the parties have learned their output, since communication between the colluding parties before they learn their recommended actions will not affect an ex ante equilibrium.) Lepinski et al. 31] show how to construct a collusion-free protocol assuming the existence of "secure envelopes"; their work is further developed in 27,28]. Some impossibility results for collusion-free protocols are shown in [31, though it is not clear what are the implications of these results for the specific problem of implementing ex ante correlated equilibria.

Collusion freeness may also be interesting in other contexts; see 3127/28] for further discussion. Recent work [27/28] has looked at stronger notions of collusion freeness, with the aim of achieving game-theoretic guarantees such as strategic equivalence between a mediated game and the cheap talk implementation of it. In that work, it is assumed that parties cannot communicate "out of band" even before the protocol begins; furthermore, a protocol should not only prevent covert communication between parties but should also prevent parties from agreeing on a common bit. We do not give further discussion here.

\subsection{Future Directions}

The immediate open question is to further characterize when a given ex post correlated equilibrium of a game is implementable (in, say, the standard communication model, either with or without broadcast). One direction to explore is when using a partially fair protocol 34171312 21 might suffice. Also, recent results [22] show that complete fairness for $t \geq n / 2$ is achievable for certain functions in the standard communication model, thus giving hope that for certain restricted classes of correlated equilibria a cheap talk implementation might be possible even when general fair computation is not. Yet another direction is to explore other communication models, e.g., when a simultaneous broadcast channel is available. Or, taking a cue from the work on collusion-free protocols, we may ask what can be achieved under the assumption that colluding parties cannot communicate once the protocol begins. (Cleve's impossibility proof [13] fails in both the aforementioned settings.) These questions are interesting both in the current context as well as in a purely cryptographic sense.

In another direction, we can strengthen Definition 13 to require cheap talk protocols to satisfy stronger game-theoretic notions such as subgame perfection. 
(See also the following section.) The Dodis-Halevi-Rabin approach, in particular, will usually not yield a subgame perfect equilibrium.

\section{Rational Multi-party Computation}

We now briefly discuss the second research direction mentioned in the Introduction. Here, there is no underlying game; rather, the protocol itself $i s$ the game, in the sense that parties' utilities are now functions of the inputs and outputs of the parties running the protocol. The difference between this setting and the standard setting of secure computation is that, in contrast to the standard setting where some parties are assumed to follow the protocol and other may behave arbitrarily, in the current setting we only guarantee that all players are rational. (Thus, the models are incomparable.) The questions here are: how can we construct "meaningful" protocols in this setting? and (more tantalizingly) does this setting enable us to circumvent impossibility results that hold under the standard definition of secure computation?

Let us jump right in with a "straw man" definition that, as far as I know, is new. Assume a set of parties $P_{1}, \ldots, P_{n}$ where party $P_{i}$ begins holding input $x_{i}$. We assume the vector of inputs $\boldsymbol{x}=\left(x_{1}, \ldots, x_{n}\right)$ is chosen according to some known distribution $\mathcal{D}$. The parties want to compute a possibly probabilistic function $f$, where $f(\boldsymbol{x})$ outputs a vector $\boldsymbol{y}=\left(y_{1}, \ldots, y_{n}\right)$ and $P_{i}$ receives $y_{i}$. The parties run some protocol $\boldsymbol{\Pi}=\left(\Pi_{1}, \ldots, \Pi_{n}\right)$, and we assume this protocol is correct in the sense that it yields the correct output if run honestly. (However, we do not assume the parties use their given inputs; see below.) The utility function of $P_{i}$ is now a polynomial-time function of its view during the execution of $\boldsymbol{\Pi}$, the initial inputs $\boldsymbol{x}$, and the outputs $\boldsymbol{y}_{-i}$ of all other parties. (Note that inputs may be viewed as types in the sense defined in Section 2.) For treating coalitions, it seems best to define, for each possible coalition $\mathcal{C}$, a utility function $u_{\mathcal{C}}$ that is a function of the coalition's view, the inputs $\boldsymbol{x}$, and the outputs $\boldsymbol{y}_{-\mathcal{C}}$ of the other parties. We let $\Gamma_{\text {real }}$ denote the real-world game thus defined.

In an ideal world computation of $f$ (see 20]), a party $P_{i}$ receiving input $x_{i}$ can replace its input with some other value $x_{i}^{\prime}=\delta_{i}\left(x_{i}\right)$; we allow $\delta_{i}$ to be probabilistic and allow $x_{i}^{\prime}=\perp$, which is treated as an abort. After parties hand their inputs to the ideal functionality, the functionality computes $\boldsymbol{y}=f\left(\boldsymbol{x}^{\prime}\right)$ and gives $y_{i}$ to $P_{i}$. Each party then outputs an arbitrary (polynomial-time) function $\pi_{i}(\cdot)$ of its view; this is left implicit in what follows, and we thus let $\delta_{i}$ stand for the entire strategy of $P_{i}$ in the ideal world game $\Gamma_{f}$. The utility functions $u_{i}$ are as above, except that these are now applied to the output of $P_{i}$, the inputs $\boldsymbol{x}$, and the outputs $\boldsymbol{y}_{-i}$ of the other parties (and analogously for coalitions).

Shoham and Tennenholtz 41] define the class of NCC functions for which, roughly speaking, setting $\delta_{i}$ to the identity function is a Nash equilibrium for all $\mathcal{D}$. Focusing on NCC functions appears to be a mistake that unnecessarily limits the class of functions under study.

Let $\Pi_{i} \circ \delta_{i}$ denote the real-world strategy where $P_{i}$ changes its input $x_{i}$ to $x_{i}^{\prime}=\delta_{i}\left(x_{i}\right)$, and then runs $\Pi_{i}$ using input $x_{i}^{\prime}$. Then: 
Definition 15. Let $\boldsymbol{\delta}=\left(\delta_{1}, \ldots, \delta_{n}\right)$ be a Nash equilibrium of $\Gamma_{f}$ with respect to utilities $\left\{u_{i}\right\}$ and input distribution $\mathcal{D}$. Then $\boldsymbol{\Pi}$ is a Nash protocol for $f$ (with respect to $\delta,\left\{u_{i}\right\}$, and $\left.\mathcal{D}\right)$ if (1) $\Pi \circ \delta=\left(\Pi_{1} \circ \delta_{1}, \ldots, \Pi_{n} \circ \delta_{n}\right)$ is a computational Nash equilibrium in $\Gamma_{\text {real }}$, and (2) for all $i$, it holds that $u_{i}(k, \boldsymbol{\Pi} \circ \boldsymbol{\delta})=u_{i}(k, \boldsymbol{\delta})$.

A definition of $t$-resilience may be derived from the above. Note that privacy, etc. are not explicitly required; it is our belief that questions of rationality should be separated from questions of security against malicious behavior.

An easy observation is that any protocol for completely fair secure computation tolerating $t$ malicious parties is a $t$-resilient protocol for any $\delta,\left\{u_{i}\right\}$, and $\mathcal{D}$. We also conjecture that if a protocol $\boldsymbol{\Pi}$ is resilient for all $\boldsymbol{\delta},\left\{u_{i}\right\}$, and $\mathcal{D}$, then it is completely fair. Thus, things only become interesting if (1) we are in a setting where completely fair secure computation is impossible; and/or (2) we look at equilibrium concepts stronger than a Nash equilibrium. We briefly discuss these issues now. More extensive discussion will appear in the full version of this paper.

Constructing Nash protocols without completely fair MPC. This relates to the question, raised earlier, as to when relying on rationality of the parties might enable circumvention of impossibility results. As one example, depending on the utilities assumed it is possible to achieve complete fairness (which, note, is attained in the ideal model used in Definition 15) even in the presence of coalitions consisting of half or more of the parties [135|24|29. Similarly, it is possible to implement Byzantine agreement over point-to-point channels even in the presence of coalitions controlling $1 / 3$ or more of the parties [23].

Rational secret sharing and stronger notions of equilibrium. Halpern and Teague [26] were the first to suggest that Nash protocols do not suffice but, instead, stronger notions are needed. As a motivating example [26, consider $t$-out-of- $n$ secret sharing (here, $t<n$ ) under the assumption that each party (1) prefers to learn the secret above all else; and (2) otherwise, prefers other parties not learn the secret. Consider the naive protocol in which each party simply broadcasts their share. (We assume authenticated shares, so each party can choose either to broadcast the correct value or nothing.) This is clearly a Nash protocol, since no matter what any particular party does at least $t$ parties broadcast their share and everyone reconstructs the secret. Nevertheless, it appears that each $P_{i}$ would prefer not to broadcast: if at least $t$ other parties broadcast, then everyone (including $P_{i}$ ) gets the secret as before; however, if fewer than $t$ parties broadcast then only $P_{i}$ recovers the secret. That is, following the protocol is weakly dominated by not following the protocol, and we might expect that no one follows the protocol. (and hence the protocol is not very useful).

To address this, Halpern and Teague suggest to look for Nash protocols where players' strategies survive iterated deletion of weakly dominated strategies. Such protocols were constructed in 26/135/24.

Kol and Naor 29] argue that the requirement of surviving iterated deletion does not suffice to rule out protocols that are, intuitively, irrational. The notion is also difficult to work with and does not seem to capture intuition very well; 
moreover, it leads to other undesirable consequences such as the fact that, if we do not assume simultaneous channels (and thus allow rushing), then protocols in which two parties are supposed to speak in the same round are inherently problematic. (Since each party will simply wait for the other to go first.) Kol and Naor thus suggest another notion that we have given as Definition 12. Their definition rules out protocols that, intuitively, seem rational to follow.

We suggest to explore using the notion of resistance to trembles. (cf. Definition 10). This requirement rules out the naive protocol mentioned above as well as the counterexample of Kol-Naor; on the other hand, the protocols of 26 13524 appear to satisfy it.

The work of 2728 offers other definitions of rational MPC.

\subsection{Future Directions}

The community has not yet settled on a definition for rational MPC, and finding the "right" definition seems important for further progress in this area. Looking at constructions, we note that almost all positive results for rational MPC thus far assume the utility functions inherited from [26] (an exception is [23]); a natural step is to characterize when rational MPC is possible for other classes of utilities. One can also look for closer connections between the questions considered in Sections 3 and 4.

More broadly, one might explore applications of the ideas described here to scenarios that are more complicated than function evaluation; trust inference in distributed systems serves as one compelling example. Another direction is to realize that secure computation does not happen in a vacuum, but instead may occur within an existing legal framework; given this, game theory might be profitably applied to analyze protocols satisfying the definitions of [5.32].

Acknowledgments. I am very grateful to Ran Canetti and the TCC '08 program committee for inviting me to write this survey, and to Ran for helpful discussions and suggestions.

\section{References}

1. Abraham, I., Dolev, D., Gonen, R., Halpern, J.: Distributed computing meets game theory: Robust mechanisms for rational secret sharing and multiparty computation. In: Proc. 25th ACM Symposium on Principles of Distributed Computing (PODC), pp. 53-62. ACM Press, New York (2006)

2. Abraham, I., Dolev, D., Halpern, J.: Lower bounds on implementing robust and resilient mediators. In: 5th Theory of Cryptography Conference (TCC) (2008), http://arxiv.org/abs/0704.3646

3. Aumann, R.: Subjectivity and correlation in randomized strategies. J. Mathematical Economics 1, 67-96 (1974)

4. Aumann, R., Hart, S.: Long cheap talk. Econometrica 71(6), 1619-1660 (2003)

5. Aumann, Y., Lindell, Y.: Security against covert adversaries: Efficient protocols for realistic adversaries. In: Vadhan, S.P. (ed.) TCC 2007. LNCS, vol. 4392, Springer, Heidelberg (2007) 
6. Barany, I.: Fair distribution protocols or how the players replace fortune. Mathematics of Operations Research 17(2), 327-340 (1992)

7. Beaver, D.: Multiparty protocols tolerating half faulty processors. In: Brassard, G. (ed.) CRYPTO 1989. LNCS, vol. 435, pp. 560-572. Springer, Heidelberg (1990)

8. Ben-Or, M., Goldwasser, S., Wigderson, A.: Completeness theorems for noncryptographic fault-tolerant distributed computation. In: 20th ACM Symposium on Theory of Computing (STOC), pp. 1-10 (1988)

9. Ben-Porath, E.: Cheap talk in games with incomplete information. J. Economic Theory 108(1), 45-71 (2003)

10. Buttyán, L., Hubaux, J.-P.: Security and Cooperation in Wireless Networks. Cambridge University Press, Cambridge (2007)

11. Chaum, D., Crépeau, C., Damgård, I.: Multiparty unconditionally secure protocols. In: 20th ACM Symposium on Theory of Computing, pp. 11-19 (1988)

12. Cleve, R.: Controlled gradual disclosure schemes for random bits and their applications. In: Brassard, G. (ed.) CRYPTO 1989. LNCS, vol. 435, Springer, Heidelberg (1990)

13. Cleve, R.: Limits on the security of coin flips when half the processors are faulty. In: 18th ACM Symposium on Theory of Computing (STOC), pp. 364-369 (1986)

14. Crawford, V., Sobel, J.: Strategic information transmission. Econometrica 50(6), 1431-1451 (1982)

15. Dodis, Y., Halevi, S., Rabin, T.: A cryptographic solution to a game theoretic problem. In: Dwork, C. (ed.) CRYPTO 2006. LNCS, vol. 4117, Springer, Heidelberg (2006)

16. Dodis, Y., Rabin, T.: Cryptography and game theory. In: Nisan, et al. [38]

17. Even, S., Goldreich, O., Lempel, A.: A randomized protocol for signing contracts. Comm. ACM 28(6), 637-647 (1985)

18. Forges, F.: Universal mechanisms. Econometrica 58(6), 1341-1364 (1990)

19. Fudenberg, D., Tirole, J.: Game Theory. MIT Press, Cambridge (1991)

20. Goldreich, O.: Foundations of Cryptography, Basic Applications, vol. 2. Cambridge University Press, Cambridge (2004)

21. Goldwasser, S., Levin, L.: Fair computation of general functions in presence of immoral majority. In: Menezes, A., Vanstone, S.A. (eds.) CRYPTO 1990. LNCS, vol. 537, pp. 77-93. Springer, Heidelberg (1991)

22. Gordon, S.D., Hazay, C., Katz, J., Lindell, Y.: Complete fairness in secure twoparty computation. Manuscript (2007)

23. Gordon, S.D., Katz, J.: Byzantine agreement with a rational adversary. In: Rump session presentation, Crypto (2006)

24. Gordon, S.D., Katz, J.: Rational secret sharing, revisited. In: Security and Cryptography for Networks (SCN), pp. 229-241 (2006)

25. Halpern, J.: Computer science and game theory: A brief survey. In: The New Palgrave Dictionary of Economics, 2nd edn. (2008), (Anticipated publication date: 2008) http://www.cs.cornell.edu/home/halpern/

26. Halpern, J., Teague, V.: Rational secret sharing and multiparty computation. In: 36th Annual ACM Symp. on Theory of Computing (STOC), pp. 623-632 (2004)

27. Izmalkov, S., Lepinski, M., Micali, S.: Rational secure computation and ideal mechanism design. In: IEEE Symposium on Foundations of Computer Science (FOCS), See also technical report MIT-CSAIL-TR-2007-040 (2005), http://hdl.handle.net/1721.1/38208

28. Izmalkov, S., Lepinski, M., Micali, S.: Verifiably secure devices. In: 5th Theory of Cryptography Conference (TCC) (2008) 
29. Kol, G., Naor, M.: Cryptography and game theory: Designing protocols for exchanging information. In: 5th Theory of Cryptography Conference (TCC) (2008)

30. Lepinski, M., Micali, S., Peikert, C., Shelat, A.: Completely fair SFE and coalitionsafe cheap talk. In: 23rd Annual ACM Symposium on Principles of Distributed Computing (PODC), pp. 1-10 (2004)

31. Lepinski, M., Micali, S., Shelat, A.: Collusion-free protocols. In: ACM Symposium on Theory of Computing (STOC) (2005)

32. Lindell, A.Y.: Legally-enforceable fairness in secure multiparty computation. In: CT-RSA (to appear 2008)

33. Linial, N.: Game-theoretic aspects of computer science. In: Aumann, R., Hart, S. (eds.) Handbook of Game Theory with Economic Applications, vol. 2, pp. 1340 1395. North-Holland, Amsterdam (1994)

34. Luby, M., Micali, S., Rackoff, C.: How to simultaneously exchange a secret bit by flipping a symmetrically-biased coin. In: 24th Annual IEEE Symposium on Foundations of Computer Science (FOCS), pp. 11-21 (1983)

35. Lysyanskaya, A., Triandopoulos, N.: Rationality and adversarial behavior in multiparty computation. In: Dwork, C. (ed.) CRYPTO 2006. LNCS, vol. 4117, Springer, Heidelberg (2006)

36. Moulin, H., Vial, J.-P.: Strategically zero sum games. Intl. J. Game Theory 7(3/4), 201-221 (1978)

37. Nash, J.: Non-cooperative games. Annals of Mathematics 54(2), 286-295 (1951)

38. Nisan, N., Roughgarden, T., Tardos, É., Vazirani, V.: Algorithmic Game Theory. Cambridge University Press, Cambridge (2007)

39. Osborne, M., Rubinstein, A.: A Course in Game Theory. MIT Press, Cambridge (2004)

40. Rabin, T., Ben-Or, M.: Verifiable secret sharing and multiparty protocols with honest majority. In: 21st ACM Symp. on Theory of Computing, pp. 73-85 (1989)

41. Shoham, Y., Tennenholtz, M.: Non-cooperative computation: Boolean functions with correctness and exclusivity. Theoretical Comp. Sci. 343(1-2), 97-113 (2005)

42. Urbano, A., Villa, J.: Computational complexity and communication: Coordination in two-player games. Econometrica 70(5), 1893-1927 (2002)

43. Urbano, A., Villa, J.: Computationally restricted unmediated talk under incomplete information. Economic Theory 23(2), 283-320 (2004) 